\title{
Baseline features and differences in 48 week clinical outcomes in patients with gastroparesis and type 1 vs type 2 diabetes
}

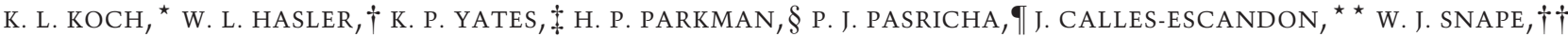

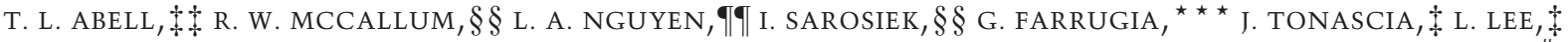 \\ L. MIRIEL $\$$ \& F. HAMILTON $\dagger \dagger \dagger$ FOR THE NIDDK GASTROPARESIS CLINICAL RESEARCH CONSORTIUM (GPCRC) ${ }^{\#}$ \\ ${ }^{\star}$ Section on Gastroenterology, Wake Forest University, Winston-Salem, NC, USA \\ $\dagger$ Division of Gastroenterology, University of Michigan, Ann Arbor, MI, USA \\ †ohns Hopkins University Bloomberg School of Public Health, Baltimore, MD, USA \\ $\S$ Section of Gastroenterology, Temple University, Philadelphia, PA, USA \\ qCenter for Neurogastroenterology, Johns Hopkins Bayview Medical Center, Baltimore, MD, USA

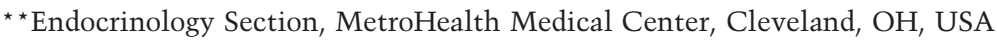 \\ $\dagger \dagger$ California Pacific Medical Center, San Francisco, CA, USA \\ țDigestive and Liver Health, University of Louisville, Louisville, KY, USA \\ $\S \S$ Division of Gastroenterology, Texas Tech University, El Paso, TX, USA \\ q $\mid$ Division of Gastroenterology, Stanford University, Palo Alto, CA, USA \\ ***Division of Gastroenterology and Hepatology, Mayo Clinic, Rochester, MN, USA \\ $\dagger \dagger$ National Institute of Diabetes and Digestive and Kidney Diseases, Bethesda, MD, USA
}

\section{Key Points}

- This study defined similarities and differences in gastroparesis severity, healthcare utilization, psychological function, and quality of life in patients with type 1 (T1DM) and type 2 (T2DM) diabetes mellitus and gastroparesis.

- At baseline enrollment into the NIDDK Gastroparesis Clinical Research Consortium Registry, T1DM patients had higher hemoglobin Alc (HbAlc) levels and more severe emptying delays, but the severity of GI symptoms was similar to those of patients with T2DM and gastroparesis.

- After 48 weeks of follow-up in the Registry, gastroparesis symptom scores significantly decreased in T2DM patients but not in T1DM patients despite increased use of prokinetic, acid suppressant, anxiolytic, and gastric electrical stimulation therapy in the T1DM group.

- Explanations for these differences in clinical outcomes at 48 weeks in patients with gastroparesis due to T1DM vs T2DM require further investigation.

Address for Correspondence

William L. Hasler, MD, University of Michigan Health System, 3912 Taubman Center, SPC 5362, Ann Arbor, MI 48109, USA.

Tel: +1 (734) 936-4780; fax: +1 (734) 936-7392;

e-mail: whasler@umich.edu

"See Appendix.

Received: 27 June 2015

Accepted for publication: 21 January 2016

\begin{abstract}
Background In studies of diabetic gastroparesis, patients with type 1 and type 2 diabetes mellitus (T1DM, T2DM) are often combined for analyses. We compared gastroparesis severity, healthcare utilization, psychological function, and quality of life in T1DM vs T2DM gastroparesis patients. Methods Questionnaire, laboratory, and scintigraphy data
\end{abstract}


from patients with gastroparesis and T1DM and T2DM from seven centers of the National Institute of Diabetes and Digestive and Kidney Diseases Gastroparesis Clinical Research Consortium Registry were compared at enrollment and after 48 weeks. Multiple regression models assessed baseline and follow-up differences between diabetes subtypes. Key Results At baseline, T1DM patients $(N=78)$ had slower gastric emptying, more hospitalizations, more gastric stimulator implantations, higher hemoglobin A1c (HbA1c), and more anxiety vs T2DM patients $(N=59)$. Independent discriminators of patients with T1DM vs T2DM included worse gastroesophageal reflux disease, less bloating, more peripheral neuropathy, and fewer comorbidities ( $p \leq 0.05)$. On follow-up, gastrointestinal (GI) symptom scores decreased only in T2DM $(p<0.05)$, but not in T1DM patients who reported greater prokinetic, proton pump inhibitor, anxiolytic, and gastric stimulator usage over 48 weeks $(p \leq 0.03)$. Gastrointestinal symptoms at baseline and 48 weeks with both subtypes were not associated with $\mathrm{HbA1c}$, peripheral neuropathy, psychological factors, or quality of life. Conclusions $\Theta$ Inferences Baseline symptoms were similar in T1DM and T2DM patients, even though T1DM patients had worse gastric emptying delays and higher HbA1c suggesting other factors mediate symptom severity. Symptom scores at 48 weeks decreased in T2DM, but not T1DM patients, despite increased medical and surgical treatment utilization by T1DM patients. Defining causes of different outcomes in diabetic gastroparesis warrants further investigation.

Keywords gastric emptying, gastroparesis, hyperglycemia, nausea and vomiting, type 1 and type 2 diabetes mellitus.

Abbreviations: BDI, Beck Depression Inventory; BMI, body mass index; CRP, C-reactive protein; ED, emergency department; ESR, erythrocyte sedimentation rate; GCSI, Gastroparesis Cardinal Symptom Index; GERD, gastroesophageal reflux disease; GES, gastric electrical stimulator; GI, gastrointestinal; GpCRC, Gastroparesis Clinical Research Consortium; HbAlc, hemoglobin A1c; NIDDK, National Institute of Diabetes and Digestive and Kidney Diseases; PAGI-QOL, Patient Assessment of Upper Gastrointestinal Disorders Quality of Life; PAGI-SYM, Patient Assessment of Upper Gastrointestinal Disorders Symptoms; SF-36v2, Short Form-36v2; STAI, State and Trait Anxiety Inventory; T1DM, type 1 diabetes mellitus; T2DM, type 2 diabetes mellitus; TPN, total parenteral nutrition.

\section{INTRODUCTION}

Diabetic gastroparesis is associated with nausea, vomiting, fullness, bloating, early satiety, and epigastric discomfort/pain and is diagnosed by documenting delayed gastric emptying. ${ }^{1-5}$ However, emptying delays correlate poorly with symptoms, suggesting other pathogenic factors influence symptoms. These factors include: (i) chronic hyperglycemia, which acutely impairs gastric neuromuscular function; (ii) gastric factors ranging from impaired fundic accommodation to gastric electrical dysrhythmias; and (iii) psychological dysfunction, which is prevalent in diabetic gastroparesis. ${ }^{6-12}$ Gastroparesis is thought to contribute to poor glycemic control which results in ketoacidosis and other complications that increase hospitalizations and outpatient visits and costs. ${ }^{13,14}$ Longitudinal studies suggest diabetic gastroparesis follows an indolent course with stable gastrointestinal (GI) symptoms and emptying rates over 25 years, although increased mortality has been reported. ${ }^{15-17}$ Furthermore, a recently published study observed no differences in overall symptom improvements over 48 weeks in patients with diabetic vs idiopathic gastroparesis. ${ }^{18}$

Type 1 diabetes mellitus (T1DM) from failed insulin production is distinct from type 2 disease (T2DM) which is due to insulin resistance and variable insulin release deficits. ${ }^{19,20}$ Type 1 diabetes mellitus requires insulin therapy, while T2DM is managed with diet and oral medications in milder cases and insulin in more severe cases. Gastroparesis is reported in $27-58 \%$ of T1DM patients vs $20-40 \%$ with T2DM; the 10 -year incidence of gastroparesis is five times higher with $\operatorname{T1DM}(5.2 \%$ vs $1.0 \%) \cdot{ }^{21-24}$ Gastroparesis is associated with increased hemoglobin A1c (HbAlc) levels and diabetic complications (retinopathy, neuropathy) in T1DM, while obesity status has been associated with symptoms in T2DM with gastroparesis. ${ }^{25,26}$ Comprehensive comparisons of clinical profiles, comorbidities, disease severity, resource utilization, psychological dysfunction, quality of life, and clinical courses in patients with gastroparesis and T1DM vs T2DM have not been performed.

Our aim was to compare the clinical features of patients with gastroparesis and T1DM and T2DM at baseline enrollment into the National Institute of Diabetes and Digestive and Kidney Diseases Gastroparesis Registry and after 48 weeks of follow-up during which time the patients' GI symptoms were managed by gastroenterologists at tertiary centers. We hypothesized that patients with T1DM gastroparesis at baseline have (i) more severe GI symptoms, (ii) more severely delayed gastric emptying, (iii) poorer glycemic 
control, (iv) more peripheral neuropathy, (v) more healthcare utilization, and (vi) more impaired psychological dysfunction and quality of life compared with patients with T2DM and gastroparesis. We further hypothesized that symptoms, psychological function, and quality of life would show similar longitudinal changes in both subtypes after 48 weeks of management.

\section{MATERIALS AND METHODS}

\section{Patient population}

Seventy-eight patients with T1DM and 59 patients with T2DM and gastroparesis in the Gastroparesis Clinical Research Consortium (GpCRC) Registry were identified. Each patient completed validated surveys and underwent examinations and blood testing on enrollment and at 48-week follow-up visits from January 2007 to May 2011 (ClinicalTrials.gov Identifier: NCT00398801). All subjects reported symptoms associated with gastroparesis for at least 12 weeks duration (not necessarily contiguous weeks) and had gastroparesis defined by scintigraphy $(>60 \%$ retention at $2 \mathrm{~h}$ and/or $>10 \%$ retention at $4 \mathrm{~h}$ ) within 6 months of enrollment. ${ }^{5}$ Prokinetics, opiates, anticholinergics, and other agents that affect gut transit were stopped at least $72 \mathrm{~h}$ before gastric emptying testing. Upper endoscopy performed within 1 year of Registry enrollment showed no evidence of organic causes of symptoms. Patients with ulcers, malignancy, mechanical obstruction, active inflammatory bowel disease, eosinophilic gastroenteritis, neurologic disease, hepatic or renal disease, other metabolic disease, or prior gastroesophageal surgery were excluded. The determination of T1DM vs T2DM status and the diagnosis of diabetic gastroparesis were made by each site investigator based upon patient reports and review of records. Studies were approved by Institutional Review Boards at each Clinical Center and Data Coordinating Center. Patients provided written informed consent.

\section{Data acquisition}

Survey completion, examinations, and local laboratory blood testing were performed on enrollment and 48-week follow-up visits. Demographic and medical information was collected on Registration and Baseline Medical History forms (Data S1), including self-reported clinician-diagnosed peripheral neuropathy. Body mass index (BMI) was calculated at both times from physical examination data; numbers and percentages who were overweight or obese $\left(\geq 25 \mathrm{~kg} / \mathrm{m}^{2}\right)$ were calculated. Numbers and percentages with any comorbidity and numbers of comorbidities were determined on enrollment (Data S1). As inflammatory activation has been identified in some cases of gastroparesis, C-reactive protein (CRP) and erythrocyte sedimentation rate (ESR) were measured on enrollment as non-specific markers of inflammation ${ }^{27}$; numbers and percentages with elevated CRP $(>0.8 \mathrm{mg} / \mathrm{dL})$ and ESR values $(>20 \mathrm{~mm} / \mathrm{h}$ ) were determined; any inflammation was defined as either an elevated CRP and/or elevated ESR. Hemoglobin Alc was quantified at both visits; numbers and percentages of patients with $\mathrm{HbAlc}$ values $<8 \%$ vs $\geq 8 \%$ were defined.

Gastroparesis severity was quantified in four ways: (i) investigator-rated gastroparesis severity was assessed on enrollment and at 48 weeks by each principal investigator using an expert consensus stratification (Supplemental Methods) ${ }^{2}$; (ii) Patient
Assessment of Upper Gastrointestinal Disorders Symptoms (PAGI-SYM) questionnaires were used to quantify 20 individual symptoms that the patient scored from 0 (none) to 5 (most severe $)^{28}$; (iii) overall symptom severity was determined by total scores from the Gastroparesis Cardinal Symptom Index (GCSI; Data $\mathrm{S} 1)^{29}$; and (iv) percentages of test meal retained at $4 \mathrm{~h}$ from pre-enrollment scintigraphy studies were used to stratify results into mild (11-20\%), moderate (21-35\%), and severe $>35 \%$ retained) gastric emptying delays. ${ }^{30}$

Medication use was queried on enrollment and at 48 weeks (Data S1). Numbers and percentages of T2DM patients taking antidiabetic medications known to cause nausea and vomiting were determined. ${ }^{25}$ Symptoms were compared in patients who were taking vs not taking these agents on enrollment.

Health utilization parameters were determined. On enrollment, patients reported how many times they were hospitalized over the prior year and for what reasons they were hospitalized. At 48 weeks, they were asked how many times they had required emergency department (ED) evaluation or hospitalization solely for gastroparesis since enrollment (excluding gastric electrical stimulator [GES] implantation). Numbers and percentages of patients on total parenteral nutrition (TPN) and who had undergone GES implantation were determined at baseline and 48 weeks.

Measures of psychological dysfunction and quality of life were quantified. Depression and anxiety were enumerated by the Beck Depression Inventory (BDI) and State and Trait Anxiety Inventory (STAI; Data S1). ${ }^{31,32}$ Numbers and percentages with severe depression (BDI score $>28$ ), state anxiety ( 11 score $\geq 50$ ), and trait anxiety (Y2 score $\geq 50$ ) were calculated. Disease-specific and generic quality of life was assessed by Patient Assessment of Upper Gastrointestinal Disorders Quality of Life (PAGI-QOL) and Short Form-36v2 (SF-36v2) surveys, respectively (Data S1).33,34

Enrollment (baseline) symptom scores were subtracted from 48-week values to calculate changes in all measures. Baseline BMI, HbAlc, hospitalizations for gastroparesis, patients on TPN or undergoing GES, BDI, Y1, Y2, and quality of life scores were subtracted from 48 -week levels to quantify changes. Numbers and percentages of patients on different medications at baseline were subtracted from 48 -week values to estimate changes.

\section{Statistical methodologies}

Number and percentages or means \pm SD were reported for enrollment categorical or continuous characteristics. $p$ values were determined from Pearson chi-squared or Fisher's exact tests for categorical characteristics and Kruskal-Wallis tests to account for non-normality of continuous distributions. ${ }^{35}$ Baseline discriminators of diabetes subtype were determined from backward stepwise multiple logistic modeling regressing diabetes subtype on the 46 baseline characteristics, forcing age at enrollment, sex, and white race into the model, with $p$ for exclusion $=0.05 .^{36}$ Total number rather than individual comorbidities was included; GCSI was excluded due to collinearity. Hosmer-Lemeshow testing revealed adequate fit for the model $(p=0.71)$. Differences between patients completing 48-week follow-up vs patients with only enrollment data were assessed using multiple logistic regression of 48-week completion on baseline characteristics (diabetes subtype, demographics, BMI, severity, medications [prokinetics, opiates, antidepressants], healthcare utilization, psychological function, quality of life). Mean changes \pm SD at 48 weeks vs enrollment were computed for all characteristics except ED visits (not queried on enrollment). For continuous characteristics, $p$ values were determined using one sample $t$-tests of the null hypothesis of no difference in means at both visits 
within diabetes subtype comparisons. For binary characteristics and medication changes, exact McNemar's tests for paired proportions were used to determine $p$ and $95 \%$ confidence intervals (CI) which were computed using continuity corrections. ${ }^{37,38}$ Multiple regression models of 48 -week changes in continuous characteristics, adjusting for enrollment values, assessed changes between diabetes subtype (except for ED visits). ${ }^{39}$ Negative binomial regressions (to account for overdispersion) of ED visits over 48 weeks on diabetes subtypes were used. Wald's tests using conditional logistic regression tested if 48-week changes in hospitalizations for gastroparesis or medication use varied by subtype. ${ }^{36}$ Unconditional exact logistic regression assessed TPN use and GES changes with T2DM. Relative odds of changes in 48-week outcomes were derived from logistic regression models of each indicator at 48 weeks in relation to subtype and enrollment value of the indicator. Models included propensity scores to adjust diabetes subtype effects for probabilities of being T1DM based on age, sex, and race. ${ }^{40}$ Outcome indicators defined by 48 -week changes from enrollment included any symptom score decrease, no change or decreased BMI, any HbAlc decrease, $\geq 5$ point BDI decrease, any STAI decrease, and any QOL increase. Healthcare utilization reductions were defined as no hospitalizations or ED visits for gastroparesis over 48 weeks.

Given the exploratory nature of our study, $p$ values were twosided and nominal with significance at the $p=0.05$ level, a priori. Because a goal of these exploratory analyses was to generate new hypotheses to be tested in future confirmatory studies, correction for multiple comparisons was not performed. Such adjustments reduce the power of an investigation to define important differences, are unnecessary if exploratory research questions are unrelated, and are only required for studies which aim to offer decisive proof of a predefined hypothesis to endorse decisionmaking protocols. ${ }^{41-43}$ Stata (Stata Statistical Software, Release v12; StataCorp LP, College Station, TX, USA) and SAS (version 9.3; SAS Institute, Inc., Cary, NC, USA) software were employed.

\section{RESULTS}

\section{Demographic and clinical factors at baseline}

Demographic and clinical factors and comorbidities for the T1DM and T2DM patients with gastroparesis at baseline are shown in Table 1. Type 2 diabetes mellitus patients with gastroparesis had several expected differences compared with T1DM patients. These T2DM patients were older at enrollment and at the onset of GI symptoms, had higher BMIs, and were more often overweight, obese, or postmenopausal $(p<0.001)$. Type 1 diabetes mellitus patients reported longer durations of diabetes prior to the onset of gastroparesis $(p=0.005)$. On average, HbAlc levels were greater in T1DM patients by $0.9 \%(p=0.003)$; T1DM patients comprised larger proportions with HbAlc levels $\geq 8 \%(37 / 53,69.8 \%)$ vs $<8 \% \quad(38 / 81$, $46.9 \% ; p=0.009)$. Almost all T1DM (98.7\%) and T2DM $(98.3 \%)$ patients reported $\geq 1$ comorbidity, but numbers of comorbidities were higher with T2DM $(5.5 \pm 3.4$ vs $4.0 \pm 2.8 ; p=0.005)$. Coronary and cerebrovascular disease and interstitial cystitis were significantly more common in T2DM patients $(p \leq 0.05)$.
Type 2 diabetes mellitus patients more often underwent hysterectomies $(p<0.001)$. Similar percentages of T1DM vs T2DM patients reported peripheral neuropathy $(43.6 \%$ vs $37.3 \%, p=0.46)$.

\section{Comparisons of gastroparesis-related factors in T1DM vs T2DM at baseline}

Gastroparesis severity, GCSI results, and resource utilization The most severe symptoms reported by both T1DM and T2DM patients with gastroparesis were nausea and postprandial fullness. Patients with T1DM and gastroparesis were more often assigned by the investigator to the severe gastroparesis category $(49 \%$ vs $39 \%)$ and less to the mild category $16 \%$ vs $16 \%)$ compared with T2DM patients $(p=0.05$; Table 2). However, patient-rated overall GCSI scores were similar in T1DM and T2DM patients $(2.8 \pm 1.1$ vs $3.0 \pm 1.0, p=0.28$ ). Individual GI symptoms were also similar, except for higher bloating symptoms in T2DM patients $(p=0.04)$. More T1DM patients had delayed emptying at $2 \mathrm{~h}(p=0.006)$ and $4 \mathrm{~h}(p<0.001)$ after ingestion of the meal, and more T1DM patients had severe emptying delays ( $>35 \%$ 4-h retention) compared with T2DM patients $154 \%$ vs $32 \%$, $p=0.001$.

Medication use from prokinetics to opiates was similar in the two subtypes, except T2DM patients had more metformin use $(p<0.001)$. Nineteen of 59 T2DM gastroparetics $(32 \%)$ used metformin on enrollment; none were on other antihyperglycemic agents (exenatide, liraglutide, pramlintide) that cause nausea. Overall, GI symptoms were similar in the T2DM patients who were taking metformin vs those patients not taking metformin $(p=0.91)$, although vomiting scores on average were lower in the group receiving metformin at baseline by 1.0 point ( $p=0.04$; Table S1).

Type 1 diabetes mellitus patients reported more hospitalizations in the year before enrollment solely for gastroparesis $(5.1 \pm 6.4$ vs $3.2 \pm 6.6, p=0.003)$, and were hospitalized more often for nausea and vomiting $(p=0.001)$, abdominal pain $(p=0.003)$, and dehydration $(p=0.01)$ compared with T2DM patients (Table 2). Total parenteral nutrition use at baseline was similar in the T1DM and T2DM patients $(p=0.78)$. More TIDM patients underwent GES implantation before enrollment in the GpCRC Registry $(15 \%$ vs $3 \%, p=0.02)$.

Psychological function and quality of life More T1DM patients with gastroparesis reported severe state anxiety (Y1 score $\geq 50 ; p=0.04$ ) and although not significant, more severe trait anxiety $(\mathrm{Y} 2$ score $\geq 50$; 
Table 1 Comparison of baseline demographic and clinical factors in T1DM vs T2DM

\begin{tabular}{|c|c|c|c|c|c|c|c|}
\hline \multirow[b]{2}{*}{ Category } & \multirow[b]{2}{*}{ Characteristic } & & \multicolumn{2}{|c|}{$\begin{array}{l}\text { Type } 1 \text { diabetics } \\
(N=78)\end{array}$} & \multicolumn{2}{|c|}{$\begin{array}{l}\text { Type } 2 \text { diabetics } \\
(N=59)\end{array}$} & \multirow[b]{2}{*}{$p$ Value $^{\star}$} \\
\hline & & & $N$ & $\%$ or mean $\pm \mathrm{SD}$ & $N$ & $\%$ or mean $\pm \mathrm{SD}$ & \\
\hline \multirow[t]{24}{*}{ Demographic/clinical } & \multirow[t]{8}{*}{ Demographic } & Female sex & 55 & $70.5 \%$ & 45 & $76.3 \%$ & 0.56 \\
\hline & & Age (years) & 78 & $39 \pm 11$ & 59 & $53 \pm 11$ & $<0.001$ \\
\hline & & White race & 60 & $76.9 \%$ & 45 & $76.3 \%$ & 1.00 \\
\hline & & Hispanic ethnicity & 7 & $9.0 \%$ & 5 & $8.5 \%$ & 1.00 \\
\hline & & Married & 42 & $53.9 \%$ & 38 & $64.4 \%$ & 0.23 \\
\hline & & College degree & 15 & $19.2 \%$ & 12 & $20.3 \%$ & 1.00 \\
\hline & & Income $>\$ 50 \mathrm{~K}$ & 36 & $46.2 \%$ & 25 & $42.4 \%$ & 0.73 \\
\hline & & Ever smoked regularly & 23 & $29.5 \%$ & 23 & $39.0 \%$ & 0.28 \\
\hline & \multirow[t]{7}{*}{ Medical History } & Age at symptom onset (years) & 78 & $34 \pm 10$ & 59 & $49 \pm 11$ & $<0.001$ \\
\hline & & $\begin{array}{l}\text { Time from diabetes onset to } \\
\text { initial symptoms (years) }\end{array}$ & 78 & $14.0 \pm 11.0$ & 59 & $8.4 \pm 8.0$ & 0.005 \\
\hline & & Symptom duration (years) & 78 & $6.2 \pm 6.3$ & 59 & $3.9 \pm 3.3$ & 0.13 \\
\hline & & Peripheral neuropathy & 34 & $43.6 \%$ & 22 & $37.3 \%$ & 0.46 \\
\hline & & Acute onset & 46 & $59.0 \%$ & 27 & $45.8 \%$ & 0.11 \\
\hline & & Initial infectious prodrome & 11 & $14.1 \%$ & 8 & $13.6 \%$ & 1.00 \\
\hline & & Postmenopausal (if female) & 12 & $21.8 \%$ & 28 & $62.2 \%$ & $<0.001$ \\
\hline & \multirow[t]{4}{*}{ Anthropometric } & BMI $\left(\mathrm{kg} / \mathrm{m}^{2}\right)$ & 78 & $26 \pm 6$ & 59 & $33 \pm 8$ & $<0.001$ \\
\hline & & Underweight & 2 & $3 \%$ & 0 & $0 \%$ & $<0.001$ \\
\hline & & Normal & 37 & $47 \%$ & 8 & $14 \%$ & \\
\hline & & Overweight or obese & 39 & $50 \%$ & 51 & $86 \%$ & \\
\hline & \multirow[t]{5}{*}{ Laboratory } & $\mathrm{CRP}(\mathrm{mg} / \mathrm{dl})$ & 78 & $0.9 \pm 1.5$ & 58 & $0.7 \pm 0.6$ & 0.10 \\
\hline & & CRP elevated $>0.8 \mathrm{mg} / \mathrm{dl}$ & 20 & $25.6 \%$ & 19 & $32.8 \%$ & 0.36 \\
\hline & & $\mathrm{ESR}(\mathrm{mm} / \mathrm{h})$ & 78 & $26 \pm 24$ & 59 & $28 \pm 25$ & 0.53 \\
\hline & & ESR elevated $>20 \mathrm{~mm} / \mathrm{h}$ & 37 & $47.4 \%$ & 30 & $50.9 \%$ & 0.69 \\
\hline & & Hemoglobin Alc (\%) & 78 & $8.3 \pm 2.0$ & 59 & $7.4 \pm 1.7$ & 0.003 \\
\hline \multirow[t]{12}{*}{ Comorbidities } & \multicolumn{2}{|c|}{ Peptic ulcer disease } & 3 & $3.9 \%$ & 5 & $8.5 \%$ & 0.29 \\
\hline & \multicolumn{2}{|c|}{ GERD } & 41 & $52.6 \%$ & 38 & $64.4 \%$ & 0.16 \\
\hline & \multicolumn{2}{|c|}{ Gallstones/gallbladder disease } & 21 & $26.9 \%$ & 25 & $42.4 \%$ & 0.06 \\
\hline & \multicolumn{2}{|c|}{ Coronary artery/cerebrovascular disease } & 6 & $7.7 \%$ & 11 & $18.6 \%$ & 0.05 \\
\hline & \multicolumn{2}{|c|}{ Endometriosis } & 4 & $5.1 \%$ & 7 & $11.9 \%$ & 0.21 \\
\hline & \multicolumn{2}{|c|}{ Interstitial cystitis } & 0 & $0.0 \%$ & 4 & $6.8 \%$ & 0.03 \\
\hline & \multicolumn{2}{|c|}{ Prior hysterectomy } & 9 & $16.4 \%$ & 22 & $48.9 \%$ & $<0.001$ \\
\hline & \multicolumn{2}{|c|}{ Migraine headaches } & 19 & $24.4 \%$ & 22 & $37.3 \%$ & 0.10 \\
\hline & \multicolumn{2}{|c|}{ Chronic fatigue syndrome } & 4 & $5.1 \%$ & 2 & $3.4 \%$ & 0.70 \\
\hline & \multicolumn{2}{|c|}{ Fibromyalgia } & 5 & $6.4 \%$ & 7 & $11.9 \%$ & 0.26 \\
\hline & \multicolumn{2}{|l|}{ Major depression } & 22 & $28.2 \%$ & 19 & $32.2 \%$ & 0.61 \\
\hline & \multicolumn{2}{|l|}{ Severe anxiety } & 8 & $10.3 \%$ & 5 & $8.5 \%$ & 0.72 \\
\hline
\end{tabular}

${ }^{\star} p$ (two-sided) determined from either a chi-squared test or Fisher's exact test for categorical characteristics or a Kruskal-Wallis test to account for non-normality of the continuous variables.

Bold values represent statistical significance $p \leq 0.05$.

$p=0.06)$ compared with T2DM patients. Other psychological survey, overall PAGI-QOL, individual PAGI-QOL domain, and SF-36v2 scores were similar in the two diabetes subtypes (Table 2).

Relationships among gastroparesis factors, HbA1c levels, and peripheral neuropathy on subgroup analysis Table 3 shows investigator-rated severity of gastroparesis and patient-scored GCSI results in the two subgroups according to baseline $\mathrm{HbAlc}$ values $<8 \%$ vs $\geq 8 \%$ and the presence or absence of peripheral neuropathy. HbAlc groupings had no relationship with the investigator ratings of gastroparesis severity, overall GCSI scores and individual GI symptom scores. Delays in gastric emptying at 2 or $4 \mathrm{~h}$ were not related to HbAlc status $(p=0.96$ or $p=0.79)$. Gastrointestinal symptom severities (except for postprandial fullness) were similar whether the T1DM and T2DM patients did or did not report peripheral neuropathy. Investigator-rated gastroparesis severity and delays in gastric emptying were similar regardless of peripheral neuropathy status.

Characteristics that discriminated patients with gastroparesis and T1DM vs T2DM at baseline Fortysix baseline predictors were used in regression analyses to determine clinical characteristics that distinguished the T1DM and T2DM patients. Few baseline characteristics discriminated the subtypes (Table 4). Compared with T2DM gastroparesis patients, T1DM patients were about one-third less likely to have more severe bloating $(\mathrm{OR}=0.62, p=0.02)$ and almost twice 
Table 2 Comparison of baseline severity, healthcare use, psychological function, and quality of life in T1DM vs T2DM between diabetic subtypes

\begin{tabular}{|c|c|c|c|c|c|c|c|}
\hline \multirow[b]{2}{*}{ Category } & \multirow[b]{2}{*}{ Characteristic } & & \multicolumn{2}{|c|}{$\begin{array}{l}\text { Type } 1 \text { diabetics } \\
(N=78)\end{array}$} & \multicolumn{2}{|c|}{$\begin{array}{l}\text { Type } 2 \text { diabetics } \\
(N=59)\end{array}$} & \multirow[b]{2}{*}{$p$ Value $^{\star}$} \\
\hline & & & $N$ & $\%$ or mean $\pm S D$ & $N$ & $\%$ or mean $\pm S D$ & \\
\hline \multirow{25}{*}{$\begin{array}{l}\text { Gastroparesis } \\
\text { severity }\end{array}$} & \multirow[t]{3}{*}{ Investigator-rated } & Grade 1 & 4 & $5.8 \%$ & 9 & $15.5 \%$ & \multirow[t]{3}{*}{0.05} \\
\hline & & Grade 2 & 36 & $46.2 \%$ & 27 & $46.6 \%$ & \\
\hline & & Grade 3 & 38 & $49.4 \%$ & 23 & $39.0 \%$ & \\
\hline & \multirow[t]{17}{*}{ Patient-rated } & Overall GCSI & 78 & $2.8 \pm 1.1$ & 59 & $3.0 \pm 1.0$ & 0.28 \\
\hline & & Nausea & 78 & $3.4 \pm 1.3$ & 59 & $3.2 \pm 1.2$ & 0.20 \\
\hline & & Retching & 78 & $2.4 \pm 1.7$ & 59 & $2.5 \pm 1.7$ & 0.99 \\
\hline & & Vomiting & 78 & $2.7 \pm 1.8$ & 59 & $2.4 \pm 1.7$ & 0.30 \\
\hline & & Stomach fullness & 78 & $3.2 \pm 1.6$ & 59 & $3.6 \pm 1.0$ & 0.27 \\
\hline & & Unable to finish meal & 78 & $2.9 \pm 1.5$ & 59 & $3.2 \pm 1.2$ & 0.43 \\
\hline & & Postprandial fullness & 78 & $3.3 \pm 1.5$ & 59 & $3.5 \pm 1.3$ & 0.80 \\
\hline & & Loss of appetite & 78 & $2.8 \pm 1.6$ & 59 & $2.8 \pm 1.4$ & 0.96 \\
\hline & & Bloating & 78 & $2.8 \pm 1.7$ & 59 & $3.4 \pm 1.4$ & 0.04 \\
\hline & & Visible distention & 78 & $2.5 \pm 1.8$ & 59 & $2.9 \pm 1.7$ & 0.19 \\
\hline & & Upper abdominal pain & 78 & $2.8 \pm 1.9$ & 59 & $2.8 \pm 1.7$ & 0.84 \\
\hline & & Upper abdominal discomfort & 78 & $2.9 \pm 1.8$ & 59 & $3.2 \pm 1.5$ & 0.47 \\
\hline & & Lower abdominal pain & 78 & $2.3 \pm 1.7$ & 59 & $1.8 \pm 1.4$ & 0.09 \\
\hline & & Lower abdominal discomfort & 78 & $2.3 \pm 1.6$ & 59 & $1.9 \pm 1.5$ & 0.15 \\
\hline & & GERD & 78 & $2.0 \pm 1.4$ & 59 & $1.9 \pm 1.3$ & 0.86 \\
\hline & & Constipation & 78 & $2.3 \pm 1.7$ & 59 & $2.4 \pm 1.6$ & 0.71 \\
\hline & & Diarrhea & 78 & $2.0 \pm 1.8$ & 59 & $1.8 \pm 1.6$ & 0.48 \\
\hline & \multirow[t]{5}{*}{ Gastric function } & 2-h retention & 78 & $71 \pm 20 \%$ & 59 & $61 \pm 22 \%$ & 0.006 \\
\hline & & 4-h retention & 78 & $47 \pm 27 \%$ & 59 & $33 \pm 24 \%$ & \multirow{4}{*}{$\begin{array}{r}<0.001 \\
0.001\end{array}$} \\
\hline & & $\begin{array}{l}\text { Mild retention } \\
\text { (11-20\% 4-h retention) }\end{array}$ & 14 & $18.0 \%$ & 26 & $44.1 \%$ & \\
\hline & & $\begin{array}{l}\text { Moderate retention } \\
(21-35 \% \text { 4-h retention })\end{array}$ & 22 & $28.2 \%$ & 14 & $23.7 \%$ & \\
\hline & & $\begin{array}{l}\text { Severe retention } \\
\text { (>35\% 4-h retention) }\end{array}$ & 42 & $53.9 \%$ & 19 & $32.2 \%$ & \\
\hline \multirow[t]{10}{*}{ Medications } & & Prokinetics & 54 & $69.2 \%$ & 38 & $64.4 \%$ & 0.55 \\
\hline & & Antiemetics & 55 & $70.5 \%$ & 40 & $67.8 \%$ & 0.73 \\
\hline & & Proton pump inhibitors/other GI agents & 62 & $79.5 \%$ & 49 & $83.1 \%$ & 0.60 \\
\hline & & NSAIDs & 42 & $53.9 \%$ & 40 & $67.8 \%$ & 0.10 \\
\hline & & Opiates & 36 & $46.2 \%$ & 28 & $47.5 \%$ & 0.88 \\
\hline & & Pain modulators & 21 & $26.9 \%$ & 17 & $28.8 \%$ & 0.81 \\
\hline & & Antidepressants & 33 & $42.3 \%$ & 21 & $35.6 \%$ & 0.43 \\
\hline & & Anxiolytics & 7 & $9.0 \%$ & 12 & $20.3 \%$ & 0.06 \\
\hline & & Antidiabetics & 74 & $94.9 \%$ & 56 & $94.9 \%$ & 1.00 \\
\hline & & Metformin & 2 & $2.6 \%$ & 19 & $32.2 \%$ & $<0.001$ \\
\hline \multirow{8}{*}{$\begin{array}{r}\text { Health care } \\
\text { utilization }\end{array}$} & \multicolumn{2}{|c|}{ Hospitalized for gastroparesis in past year } & 57 & $73.1 \%$ & 27 & $45.8 \%$ & 0.001 \\
\hline & \multirow{2}{*}{\multicolumn{2}{|c|}{ Number of hospitalizations for gastroparesis in past year }} & 78 & $5.1 \pm 6.4$ & 59 & $3.2 \pm 6.6$ & 0.003 \\
\hline & & Nausea/vomiting & 54 & $72.0 \%$ & 25 & $43.9 \%$ & 0.001 \\
\hline & & Abdominal pain & 33 & $61.1 \%$ & 15 & $31.9 \%$ & 0.003 \\
\hline & & Dehydration & 39 & $65.0 \%$ & 22 & $40.7 \%$ & 0.01 \\
\hline & & GI hemorrhage & 6 & $22.2 \%$ & 3 & $8.6 \%$ & 0.16 \\
\hline & On TPN & & 8 & $10.3 \%$ & 5 & $8.5 \%$ & 0.78 \\
\hline & Underwent GES & & 12 & $15.4 \%$ & 2 & $3.4 \%$ & 0.02 \\
\hline \multirow{6}{*}{$\begin{array}{l}\text { Psychological } \\
\text { function }\end{array}$} & BDI & & 78 & $22 \pm 13$ & 59 & $19 \pm 10$ & 0.78 \\
\hline & BDI score $>28$ & & 20 & $25.6 \%$ & 10 & $17.0 \%$ & 0.22 \\
\hline & Y1 state anxiety & & 78 & $48 \pm 14$ & 59 & $45 \pm 13$ & 0.19 \\
\hline & Y1 score $\geq 50$ & & 37 & $47.4 \%$ & 18 & $30.5 \%$ & 0.04 \\
\hline & Y2 trait anxiety & & 78 & $47 \pm 13$ & 59 & $44 \pm 13$ & 0.08 \\
\hline & Y2 score $\geq 50$ & & 35 & $44.9 \%$ & 17 & $28.8 \%$ & 0.06 \\
\hline Quality & Overall PAGI-QOL & & 78 & $2.4 \pm 1.1$ & 59 & $2.6 \pm 1.2$ & 0.23 \\
\hline of life & PAGI-QOL subscore & Daily activities & 78 & $2.2 \pm 1.3$ & 59 & $2.4 \pm 1.2$ & 0.21 \\
\hline & & Clothing & 78 & $3.0 \pm 1.7$ & 59 & $3.0 \pm 1.7$ & 1.00 \\
\hline & & Diet & 78 & $1.7 \pm 1.2$ & 59 & $1.8 \pm 1.3$ & 0.71 \\
\hline & & Relationship & 78 & $2.8 \pm 1.7$ & 59 & $3.1 \pm 1.5$ & 0.28 \\
\hline & & Psychological & 78 & $2.4 \pm 1.5$ & 59 & $2.8 \pm 1.4$ & 0.08 \\
\hline & SF-36v2 physical & & 78 & $33 \pm 10$ & 59 & $30 \pm 9$ & 0.11 \\
\hline & SF-36v2 mental & & 78 & $34 \pm 12$ & 59 & $37 \pm 13$ & 0.16 \\
\hline
\end{tabular}

${ }^{*} p$ (two-sided) determined from either a chi-squared test or Fisher's exact test for categorical characteristics or a Kruskal-Wallis test to account for non-normality of the continuous variables.

Bold values represent statistical significance $p \leq 0.05$. 


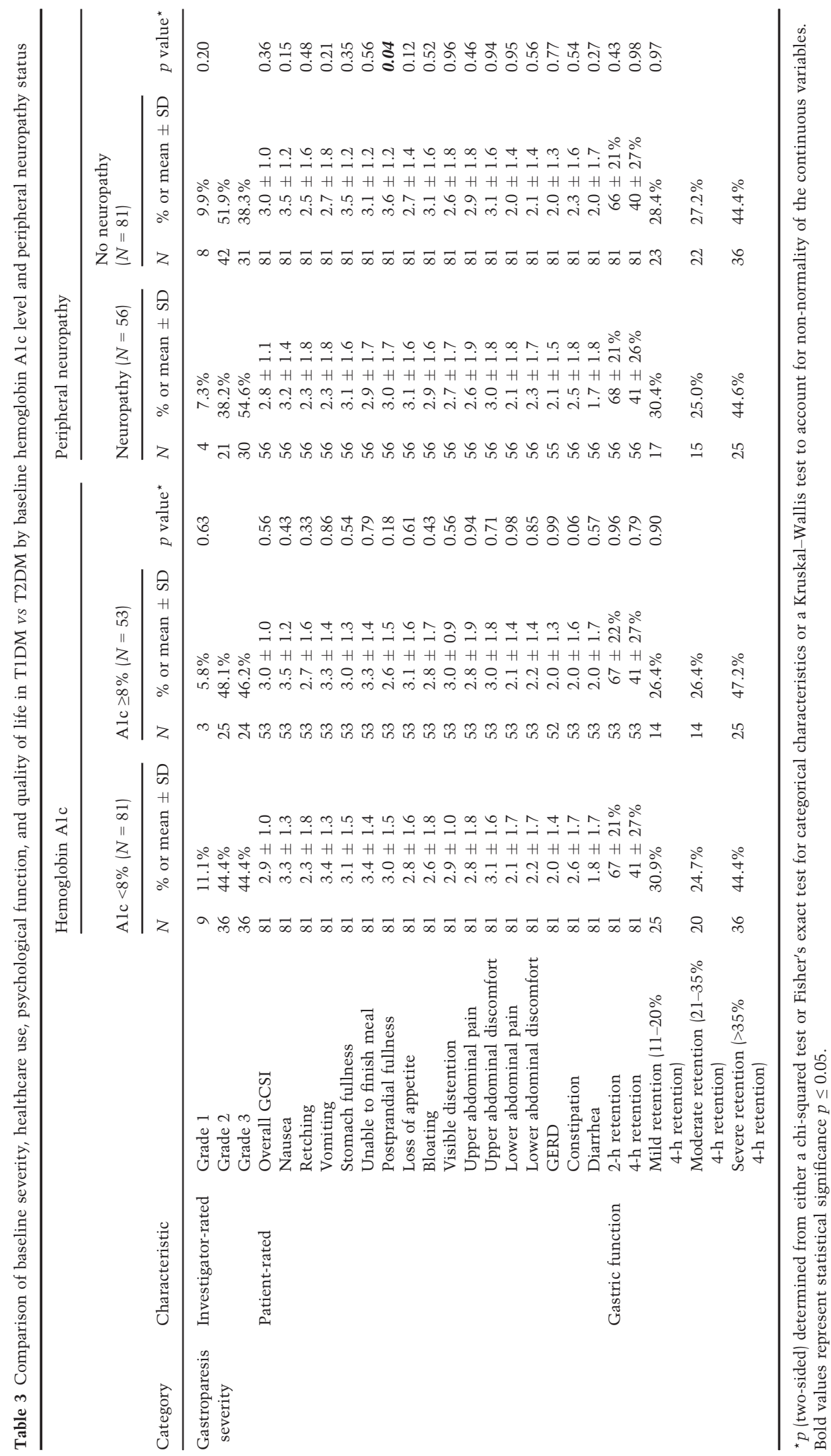


Table 4 Baseline clinical discriminators of diabetes subtype (T1DM vs T2DM) in patients with gastroparesis

\begin{tabular}{|c|c|c|c|c|}
\hline Category & Characteristic & $\mathrm{OR}^{\star}$ & $95 \% \mathrm{CI}$ & $p$ value $^{\dagger}$ \\
\hline \multirow[t]{3}{*}{ Demographic } & Age $(\geq 50$ vs $<50$ years $)$ & 0.07 & $0.02,0.24$ & $<0.001$ \\
\hline & Sex (female vs male) & 1.39 & $0.47,4.07$ & 0.17 \\
\hline & Race (white vs non-white) & 2.78 & $0.84,9.20$ & 0.40 \\
\hline \multirow[t]{3}{*}{ Clinical } & Peripheral neuropathy (yes $v s$ no) & 3.81 & $1.28,11.35$ & 0.02 \\
\hline & Overweight or obese $\left(\mathrm{BMI} \geq 25 \mathrm{vs}<25 \mathrm{~kg} / \mathrm{m}^{2}\right)$ & 0.11 & $0.04,0.36$ & $<0.001$ \\
\hline & Number of comorbidities & 0.76 & $0.63,0.92$ & 0.02 \\
\hline \multirow[t]{2}{*}{ Symptom severity } & Bloating & 0.62 & $0.42,0.91$ & 0.02 \\
\hline & GERD & 1.70 & $1.08,2.67$ & 0.02 \\
\hline
\end{tabular}

*A total of 136 diabetic patients with delayed emptying were included in the analysis: T1DM = 78, T2DM = 58; one T2DM patient has missing data for the GERD subscale on enrollment. ${ }^{\dagger} p$ derived from a backward stepwise multiple logistic model regressing diabetes subtype (T1DM vs T2DM on the candidate set of 46 baseline predictors included in Tables 1 and 2 ( $p$ for exclusion $=0.05$ ), where age, sex, and white race were forced into the model. GCSI was not included due to collinearity with its components. One measure of inflammation was used (either a high CRP and/or a high ESR). Total numbers of comorbidities were included instead of individual comorbidities. A two category symptom duration ( $\geq 5$ vs $<5$ years) was used due to small numbers in the categories. Final model is presented. Hosmer-Lemeshow Goodness of fit $\chi^{2}(\mathrm{df}=86)=3.77, p=0.71$.

Bold values represent statistical significance $p \leq 0.05$.

as likely to have gastroesophageal reflux disease (GERD) symptoms (OR $=1.70, p=0.02)$. Type 1 diabetes mellitus patients were younger $\left(\mathrm{OR}_{\mathrm{Age} \geq 50 \mathrm{yrs}}=0.07, p<0.001\right)$, had more peripheral neuropathy $(\mathrm{OR}=3.81, p=0.02)$, had more than nine times the odds of normal or underweight status $(\mathrm{OR}=0.11, p<0.001)$, and reported approximately $25 \%$ fewer numbers of comorbidities $(\mathrm{OR}=0.76$, $p=0.02)$ than T2DM patients with gastroparesis.

\section{Clinical factors at 48 weeks}

Ninety of 137 enrolled patients (66\%) completed the 48-week visit: 44 patients with T1DM (56\% of baseline cohort) and 46 patients with T2DM $(79 \%$ of the baseline cohort). Compared to those with only enrollment data, patients completing follow-up were more often male, white race, and overweight and less likely to have GES surgery $(p \leq 0.05)$; diabetic subgroup was not associated with completing follow-up with adjustment for all other characteristics (OR T1DM vs $\mathrm{T} 2 \mathrm{DM}=0.35, p=0.09$; Table S2).

Body mass index did not change significantly over the 48 weeks in these T1DM and T2DM patients as shown in Table 5. Hemoglobin Alc levels increased similarly, but not significantly, compared with baseline over the 48 weeks in both T1DM and T2DM patients $(p=0.51)$. Three patients with T1DM and one with T2DM died during the 48-week period.

\section{Comparisons of gastroparesis-related factors in T1DM vs T2DM at 48 weeks}

Gastroparesis severity, GCSI results, and resource utilization Gastrointestinal symptom severity did not decrease at 48 weeks in the patients with T1DM as measured by GCSI and individual scores (Fig. 1A). In contrast, overall GCSI scores and all individual symptoms (except postprandial fullness and visible distention) decreased significantly at 48 weeks in the T2DM patients (Fig. 1B). Fig. 1C shows the changes in patient-reported symptoms $( \pm 95 \% \mathrm{CI})$ at 48 weeks for both subtypes. Investigator-rated gastroparesis severity ratings showed similar reductions from baseline within T1DM (mean change $=-0.33, p=0.009$ ) and T2DM (mean change $=-0.30, p=0.02$ ) patients; however, these changes were not different between the subtypes $\left(\mathrm{P}_{\text {TIDM vs T2DM }}=0.23\right.$; Table 5$)$.

Increased use of prokinetic drugs $(+15.9 \%)$, proton pump inhibitor/other GI agents $(+13.6 \%)$, and anxiolytic drugs $(+25.0 \%)$ was recorded in T1DM patients $(p \leq 0.03)$, whereas increased use of opiates $(+17.4 \%)$ was documented in T2DM patients $(p=0.04)$ at 48 weeks compared with baseline (Table 5). Percentages of patients hospitalized for gastroparesis during the 48 -week follow-up decreased $15.1 \%$ in the T1DM patients $(p=0.04)$, but did not significantly change for T2DM patients; however, decreases in hospitalizations for T1DM vs T2DM patients were not significantly different $(p=0.26)$. The number of ED visits and changes in TPN use over 48 weeks were not different between diabetes subtypes. Implantation of GES devices increased $20.5 \%$ over 48 weeks in patients with T1DM $(p=0.01)$ and increased $10.9 \%$ in T2DM patients $(p=0.06)$. Including those who were implanted before enrollment, more T1DM patients were receiving GES after 48 weeks of follow-up compared with patients with T2DM and gastroparesis $(31.9 \%$ vs $10.9 \% ; p=0.02)$.

Psychological function and quality of life No changes in any psychological or quality of life parameter in either subtype or between subtypes were observed at 48 weeks of follow-up (Table 5). 
Table 5 Comparison of 48 week changes in severity, healthcare use, psychological function, and quality of life in T1DM vs T2DM

\begin{tabular}{|c|c|c|c|c|c|c|c|c|c|c|}
\hline \multirow[b]{2}{*}{ Category } & \multirow[b]{2}{*}{ Characteristic } & \multicolumn{4}{|c|}{ Type 1 diabetics $\left(N=44^{\star}\right)$} & \multicolumn{4}{|c|}{ Type 2 diabetics $\left(N=46^{*}\right)$} & \multirow[b]{2}{*}{$\begin{array}{l}\text { T1DM } \\
\text { vs T2DM } \\
p \text {-value }\end{array}$} \\
\hline & & $\begin{array}{l}\text { Mean } \\
\text { baseline }\end{array}$ & $\begin{array}{l}\text { Mean } \Delta \\
\text { ( } 48 \text { weeks } \\
\text { vs baseline) }\end{array}$ & $95 \% \mathrm{CI}$ & $p$ value $^{\dagger}$ & $\begin{array}{l}\text { Mean } \\
\text { baseline }\end{array}$ & $\begin{array}{l}\text { Mean } \Delta \\
\text { (48 weeks } \\
\text { vs baseline) }\end{array}$ & $95 \%$ CI & $p$ value $^{\dagger}$ & \\
\hline \multirow[t]{2}{*}{ Clinical } & BMI $\left(\mathrm{kg} / \mathrm{m}^{2}\right)$ & 27.2 & 0.67 & $-0.18,1.53$ & 0.12 & 34.1 & 0.55 & $-0.32,1.42$ & 0.21 & 0.44 \\
\hline & $\begin{array}{l}\text { Hemoglobin } \\
\text { Alc }(\%)\end{array}$ & 8.1 & 0.47 & $-0.32,1.27$ & 0.23 & 7.2 & 0.44 & $-0.03,0.92$ & 0.07 & 0.51 \\
\hline $\begin{array}{l}\text { Gastroparesis } \\
\text { severity }\end{array}$ & Investigator-rated & 2.5 & -0.33 & $-0.57,-0.09$ & 0.009 & 2.2 & -0.30 & $-0.55,-0.05$ & 0.02 & 0.23 \\
\hline \multirow[t]{10}{*}{ Medications } & Prokinetics & $70.5 \%$ & $15.9 \%$ & $2.8,30.0 \%$ & 0.01 & $71.7 \%$ & $8.7 \%$ & $-3.6,21.0 \%$ & 0.22 & 1.00 \\
\hline & Antiemetics & $70.5 \%$ & $4.5 \%$ & $-13.1,22.2 \%$ & 0.77 & $67.4 \%$ & $4.3 \%$ & $-13.7,22.4 \%$ & 0.79 & 0.95 \\
\hline & $\begin{array}{l}\text { Proton pump } \\
\text { inhibitors/other } \\
\text { GI agents }\end{array}$ & $81.8 \%$ & $13.6 \%$ & $1.2,26.0 \%$ & 0.03 & $80.4 \%$ & $4.3 \%$ & $-6.3,15.0 \%$ & 0.63 & 1.00 \\
\hline & NSAIDs & $52.3 \%$ & $2.3 \%$ & $-21.8,17.2 \%$ & 1.00 & $76.1 \%$ & $-17.4 \%$ & $-35.9,1.1 \%$ & 0.08 & 0.21 \\
\hline & Opiates & $47.7 \%$ & $13.6 \%$ & $-2.1,29.4 \%$ & 0.11 & $43.5 \%$ & $17.4 \%$ & $1.3,33.4 \%$ & 0.04 & 0.84 \\
\hline & Pain modulators & $29.6 \%$ & $4.5 \%$ & $-10.3,19.3 \%$ & 0.73 & $34.8 \%$ & $-2.2 \%$ & $-13.9,9.5 \%$ & 1.00 & 0.43 \\
\hline & Antidepressants & $40.9 \%$ & $9.1 \%$ & $-8.4,26.6 \%$ & 0.39 & $39.1 \%$ & $13.1 \%$ & $-5.8,31.8 \%$ & 0.21 & 0.91 \\
\hline & Anxiolytics & $15.9 \%$ & $25.0 \%$ & $9.9,40.1 \%$ & 0.001 & $21.7 \%$ & $-2.1 \%$ & $-17.1,12.8 \%$ & 1.00 & 1.00 \\
\hline & Antidiabetics & $93.2 \%$ & $0 \%$ & $-2.3,2.3 \%$ & 1.00 & $95.3 \%$ & $-2.2 \%$ & $-13.9,9.5 \%$ & 1.00 & 1.00 \\
\hline & Metformin & $2.3 \%$ & $-2.3 \%$ & $-8.9,4.4 \%$ & 1.00 & $32.6 \%$ & $-2.2 \%$ & $-16.0,11.3 \%$ & 1.00 & 1.00 \\
\hline \multirow[t]{5}{*}{$\begin{array}{r}\text { Health care } \\
\text { utilization }\end{array}$} & $\begin{array}{l}\text { Hospitalization } \\
\text { in past year }(\%)\end{array}$ & $70.5 \%$ & $-15.1 \%$ & $-30.6,-1.1 \%$ & 0.04 & $43.5 \%$ & $-8.7 \%$ & $-25.4,8.0 \%$ & 0.39 & 0.26 \\
\hline & $\begin{array}{l}\text { Number of } \\
\text { hospitalizations } \\
\text { for gastroparesis }\end{array}$ & 4.8 & -1.55 & $-3.16,0.07$ & 0.06 & 2.4 & -0.74 & $-2.22,0.74$ & 0.32 & 0.70 \\
\hline & $\begin{array}{l}\text { Number of } \\
\text { ED visits }\end{array}$ & NA & 4.86 & $2.78,6.95$ & NA & NA & 2.46 & $1.04,3.88$ & NA & 0.07 \\
\hline & $\%$ on TPN & $4.5 \%$ & $2.3 \%$ & $-7.7,12.2 \%$ & 1.00 & $8.7 \%$ & $-8.7 \%$ & $-19.0,-1.6 \%$ & 0.13 & 0.13 \\
\hline & $\begin{array}{l}\% \text { undergoing } \\
\text { GES }\end{array}$ & $11.4 \%$ & $20.5 \%$ & $4.73,36.2 \%$ & 0.01 & $0.0 \%$ & $10.9 \%$ & $-0.3,22.0 \%$ & 0.06 & 0.16 \\
\hline \multirow{3}{*}{$\begin{array}{l}\text { Psychological } \\
\text { function }\end{array}$} & $\mathrm{BDI}$ & 21.3 & -1.18 & $-4.91,2.56$ & 0.53 & 18.8 & 0.51 & $-2.42,3.44$ & 0.73 & 0.69 \\
\hline & Y1 state anxiety & 44.4 & 0.85 & $-3.96,5.66$ & 0.72 & 44.4 & 0.64 & $-3.70,4.99$ & 0.77 & 0.97 \\
\hline & $\mathrm{Y} 2$ trait anxiety & 44.1 & 2.20 & $-1.80,6.20$ & 0.27 & 44.0 & 0.58 & $-3.26,4.42$ & 0.77 & 0.53 \\
\hline \multirow{3}{*}{$\begin{array}{l}\text { Quality } \\
\text { of life }\end{array}$} & PAGI-QOL & 2.5 & 0.22 & $-0.14,0.58$ & 0.22 & 2.6 & 0.15 & $-0.11,0.42$ & 0.25 & 0.84 \\
\hline & SF-36v2 physical & 32.6 & 1.28 & $-1.72,4.27$ & 0.39 & 29.7 & 1.82 & $-0.52,4.16$ & 0.12 & 0.80 \\
\hline & SF-36v2 mental & 35.3 & 1.55 & $-2.95,6.05$ & 0.49 & 36.6 & 0.65 & $-2.96,4.26$ & 0.72 & 0.86 \\
\hline
\end{tabular}

* $N$ determined by value for medication or outcome being available at enrollment and at 48 weeks; for T1DM, between 31 and 44 ; for T2DM, between 36 and $46 .{ }^{\dagger}$ Mean change of outcome or \% medication use (48 weeks-baseline) or mean number of events in 48 weeks for ED visits, since baseline for ED visits unavailable. For continuous outcomes, $p$ value determined using one sample $t$-test of the null hypothesis of no difference in means at follow-up and baseline. For binary outcomes (hospitalized, TPN, GES) and medication use, an exact McNemar's test for paired proportions was used to determine $p$, and $95 \%$ confidence intervals (CI) determined using a continuity correction. ${ }^{*} p$ values for continuous outcomes determined using multiple regression of each outcome in relation to diabetes subtype with adjustment for the baseline value of the outcome. $p$ value for ED visits was determined using a negative binomial with robust variance to account for overdispersion. $p$ values for binary outcomes and medication use were derived from Wald tests to assess whether change in medication use varied by diabetes subtype using conditional logistic regression. Unconditional exact logistic regression was used to assess changes in TPN use or GES implantation, since no T2DM patients had those treatments at either enrollment (GES) or 48 weeks (TPN). ${ }^{\S}$ Total hospitalizations for gastroparesis since baseline exclude GES placement. NA was defined as not applicable (the number of ED visits in the past year was not queried at baseline).

Bold values represent statistical significance $p \leq 0.05$.

Factors associated with changes in gastroparesisrelated outcomes at 48 -weeks Multiple regression analyses were used to assess the relationship of eight clinical factors to outcomes at 48 weeks in the T1DM and T2DM groups with gastroparesis (Table 6). T1DM patients were less likely to report decreased vomiting $(\mathrm{OR}=0.21,95 \%$ CI: $0.05-0.87 ; p=0.03)$, but more likely to have reductions in loss of appetite scores $(\mathrm{OR}=4.25,95 \%$ CI: $1.07-16.92 ; p=0.04)$ compared to T2DM patients. When data from both diabetic subtypes were pooled, no reduction in any parameter of gastroparesis severity was related to initial $\mathrm{HbAlc}$ levels or presence of peripheral neuropathy on enrollment (Table S3). Except for decreases in abdominal pain scores in T2DM patients whose HbAlc increased over 48 weeks $(p=0.02)$, changes in symptom severity over 48 weeks were similar in T1DM and T2DM patients whose HbAlc levels either worsened or decreased (Table S4). Reduction or no reduction in $\mathrm{HbAlc}$ levels did not vary significantly between diabetic subgroups over the 48-week period (data not shown). 
A Type 1 diabetics gastroparesis symptom severity

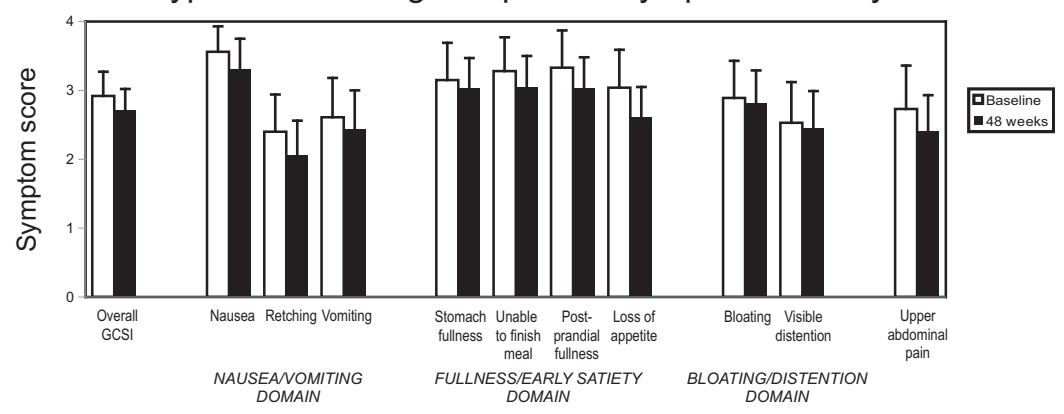

B

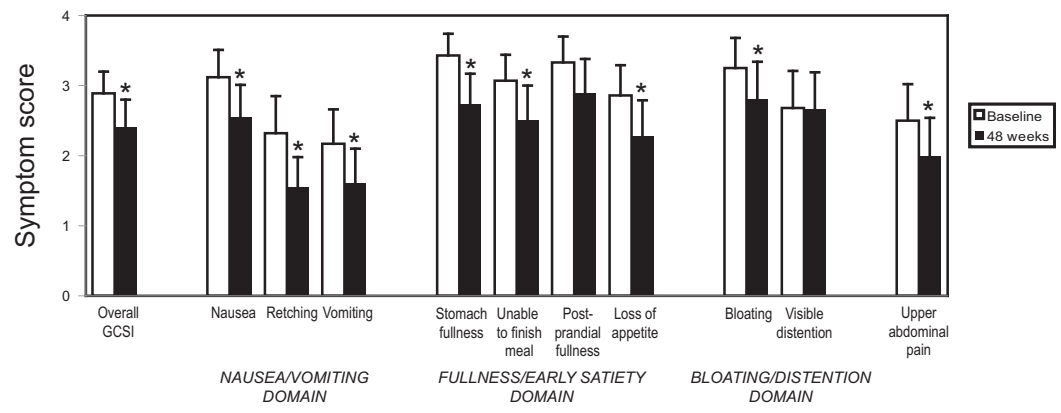

C Change in symptom severity over 48 weeks
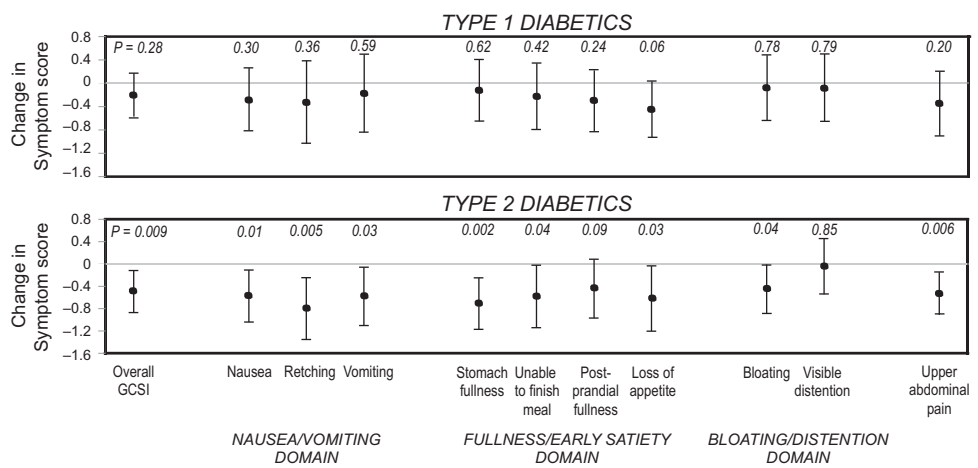

Figure 1 Patient-rated symptom scores at baseline and at 48-week follow-up. (A) T1DM patients exhibited moderate to severe baseline symptom severities (clear bars; data expressed as mean \pm upper limit of $95 \%$ CI). Symptom scores did not decrease over 48 weeks (dark bars). (B) T2DM patients exhibited baseline symptom severities in the moderate to severe range (clear bars). However, a reduction in symptoms was observed at 48 weeks for all symptoms except postprandial fullness and visible distention (dark bars). (C) Mean changes in symptoms $\pm 95 \%$ CI are plotted with $p$ values for baseline vs 48 -week values for T1DM and T2DM patients. No symptom changed significantly for T1DM patients. Overall symptom scores decreased and 8 of 10 individual symptoms significantly decreased in T2DM gastroparetics at 48week follow-up (all $p \leq 0.05$ ). "means $p \leq 0.05$.

\section{DISCUSSION}

Our findings delineate many clinical similarities in patients with T1DM and T2DM and gastroparesis and confirm several demographic differences. Gastrointestinal symptoms rated at baseline were remarkably similar in intensity between diabetic subtypes, including nausea and stomach fullness, with only greater bloating in T2DM patients and increased GERD in T1DM patients being significantly different. Our results also showed the HbAlc levels and the severity of gastric emptying delay did not correlate with the symptoms associated with gastroparesis in either patients with T1DM or T2DM; even though gastric retention severity was higher in T1DM, symptoms were not correspondingly increased at enrollment.
The poor relation of symptom severity to gastric emptying in T1DM vs T2DM patients is consistent with recent literature, and suggests other pathophysiologic abnormalities mediate GI symptom genesis. ${ }^{8,44}$ Factors such as poor fundic accommodation, heightened sensitivity to gastric distention, gastric dysrhythmias, and pyloric dysfunction warrant study as potential causes of GI symptoms associated with diabetic gastroparesis. ${ }^{45-47}$

Despite reporting similar GI symptom intensity as T2DM patients, hospitalizations for gastroparesis and for GES implantations were higher in T1DM patients at baseline. It is likely that factors other than GI symptoms such as poor glycemic control, and dehydration and electrolyte disturbances brought on by 
Table 6 Relative odds of change in clinical 48 week outcomes in relation to diabetes subtype (T1DM vs T2DM) in patients with gastroparesis

\begin{tabular}{|c|c|c|c|c|c|c|c|}
\hline Category & Change at 48 weeks from ba & eline $^{\star}$ & $\begin{array}{l}\text { Type } 1 \\
\text { diabetics, } \\
N \text { (\% improved) }\end{array}$ & $\begin{array}{l}\text { Type } \\
2 \text { diabetics, } \\
N \text { (\% improved) }\end{array}$ & $\mathrm{OR}^{\dagger}$ & $95 \%$ CI & $p$ value $e^{\ddagger}$ \\
\hline \multirow{4}{*}{$\begin{array}{l}\text { Clinical } \\
\text { measures } \\
\text { Gastroparesis } \\
\text { severity }\end{array}$} & \multicolumn{2}{|c|}{ BMI $\left(\mathrm{kg} / \mathrm{m}^{2}\right)$ (same or lower vs higher BMI) } & $42(31 \%)$ & $44(27 \%)$ & 1.86 & $0.51,6.80$ & 0.35 \\
\hline & \multicolumn{2}{|c|}{ Hemoglobin Alc (\%) (any decrease) } & $28(25 \%)$ & $36(42 \%)$ & 0.80 & $0.19,3.30$ & 0.76 \\
\hline & \multicolumn{2}{|c|}{ Investigator-rated severity ( $\geq 1$ point decrease) } & $43(33 \%)$ & $46(37 \%)$ & 0.68 & $0.21,2.13$ & 0.50 \\
\hline & \multirow[t]{11}{*}{ Patient-rated (any decrease) } & Overall GCSI & $40(60 \%)$ & $45(62 \%)$ & 1.10 & $0.32,3.71$ & 0.88 \\
\hline & & Nausea & $40(35 \%)$ & $45(51 \%)$ & 0.54 & $0.18,1.64$ & 0.28 \\
\hline & & Retching & $40(48 \%)$ & $45(56 \%)$ & 0.45 & $0.10,2.10$ & 0.31 \\
\hline & & Vomiting & $40(33 \%)$ & $45(53 \%)$ & 0.21 & $0.05,0.87$ & 0.03 \\
\hline & & Stomach fullness & $40(40 \%)$ & $45(44 \%)$ & 1.71 & $0.53,5.53$ & 0.37 \\
\hline & & Unable to finish meal & $40(35 \%)$ & $45(42 \%)$ & 1.13 & $0.33,3.81$ & 0.85 \\
\hline & & Postprandial fullness & $40(48 \%)$ & $45(40 \%)$ & 2.65 & $0.78,9.02$ & 0.12 \\
\hline & & Loss of appetite & $40(50 \%)$ & $45(42 \%)$ & 4.25 & $1.07,16.92$ & 0.04 \\
\hline & & Bloating & $40(38 \%)$ & $45(40 \%)$ & 1.68 & $0.50,5.63$ & 0.40 \\
\hline & & Visible distention & $40(30 \%)$ & $45(36 \%)$ & 1.15 & $0.32,4.13$ & 0.83 \\
\hline & & Upper abdominal pain & $40(38 \%)$ & $45(47 \%)$ & 1.81 & $0.48,6.75$ & 0.38 \\
\hline \multirow[t]{2}{*}{$\begin{array}{r}\text { Health care } \\
\text { utilization }\end{array}$} & \multicolumn{2}{|c|}{$\begin{array}{l}\text { Hospitalizations for gastroparesis (none over } 48 \text { weeks } \\
\text { VS any) }\end{array}$} & $44(45 \%)$ & $46(65 \%)$ & 0.85 & $0.26,2.77$ & 0.78 \\
\hline & \multicolumn{2}{|c|}{ ED visits (none over 48 weeks vs any) } & $44(27 \%)$ & $46(50 \%)$ & 0.43 & $0.14,1.29$ & 0.13 \\
\hline \multirow{3}{*}{$\begin{array}{l}\text { Psychological } \\
\text { function }\end{array}$} & \multicolumn{2}{|c|}{$\mathrm{BDI}(\leq 5$ point decrease) } & $40(35 \%)$ & $45(40 \%)$ & 0.91 & $0.28,2.90$ & 0.87 \\
\hline & \multirow{2}{*}{\multicolumn{2}{|c|}{$\begin{array}{l}\text { Y1 state anxiety (any decrease) } \\
\text { Y2 trait anxiety (any decrease) }\end{array}$}} & $40(45 \%)$ & $45(49 \%)$ & 0.85 & $0.25,2.95$ & 0.80 \\
\hline & & & $40(38 \%)$ & $45(49 \%)$ & 0.45 & $0.14,1.44$ & 0.18 \\
\hline \multirow{3}{*}{$\begin{array}{l}\text { Quality } \\
\text { of life }\end{array}$} & \multicolumn{2}{|l|}{ PAGI-QOL (any increase) } & $39(51 \%)$ & $43(56 \%)$ & 0.87 & $0.29,2.54$ & 0.80 \\
\hline & \multirow{2}{*}{\multicolumn{2}{|c|}{ SF-36v2 physical ( $\geq 1$ point increase) }} & $40(50 \%)$ & $43(53 \%)$ & 1.70 & $0.50,5.81$ & 0.40 \\
\hline & & & $40(53 \%)$ & $43(51 \%)$ & 1.19 & $0.39,3.66$ & 0.76 \\
\hline
\end{tabular}

*Each outcome indicator is defined as change in the characteristic score at 48 weeks compared to baseline. Outcome indicators based on change in value at 48 weeks from value at enrollment included: symptom score reduction (any decrease in total GCSI or in individual GCSI scores), BMI (same or lower), hemoglobin A1c (any decrease), psychological function (BDI decreased by 5 or more points; Y1 state-anxiety, Y2 trait-anxiety any decrease), and QOL (PAGI-QOL any increase; SF-36v2 physical and SF-36v2 mental components, increase in at least one point). ${ }^{\dagger} \mathrm{OR}=\mathrm{Relative}$ odds of change in T1DM vs T2DM. "OR's derived from logistic regression models of each indicator of change in characteristic score at 48 weeks in relation to diabetes type and baseline value of the indicator. Models included a propensity score to adjust the diabetes type effect for the probability of being T1DM based on age, sex, and race (white vs not white).

Bold values represent statistical significance $p \leq 0.05$.

acute vomiting may be more relevant drivers of hospitalizations in T1DM patients. Despite this greater resource use in T1DM, overall medication use profiles and quality of life scores were similar to T2DM gastroparetics at baseline.

The clinical perception that patients with T1DM and gastroparesis are frequently underweight is not supported by our findings. We found almost half of T1DM patients with gastroparesis were overweight or obese and only $3 \%$ were underweight, while T2DM patients with gastroparesis were even heavier as in prior reports. ${ }^{19,20}$ Baseline TPN use was noted by less than $10 \%$ of patients in both subgroups reflecting the ability of most patients to sustain intake by oral or enteral routes. However, these findings do not rule out significant nutritional impairments. Our group previously reported mean daily caloric intakes of less than 1200 calories with deficiencies in essential nutrients including vitamin $\mathrm{B}_{6}$, vitamin $\mathrm{K}$, and iron in patients with gastroparesis. ${ }^{48}$

An infectious prodrome was noted in 14\% of T1DM and T2DM patients. A similar incidence of infectious prodrome has been observed with idiopathic gastro- paresis, suggestive of a potential viral etiology in these non-diabetic patients. ${ }^{8}$ The role of infections as cofactors in triggering the onset of diabetic gastroparesis could be the focus of additional study.

A new finding of this investigation is the difference in GI symptoms in the two groups at the 48-week follow-up visits. Symptom scores decreased only in the T2DM patients while symptom severity was mostly unchanged in those with T1DM. It is possible the lack of reduction in GI symptom scores at 48 weeks in T1DM patients may reflect irreversible diabetesrelated damage to the stomach wall. However, in ultrastructural studies from full-thickness gastric biopsies, no differences were observed in the loss of enteric neurons, depletion of interstitial cells of Cajal, or in myenteric immune cell infiltration in specimens from T1DM vs T2DM patients. ${ }^{49}$ This differential outcome in GI symptoms in T1DM vs T2DM patients occurred despite aggressive management over the 48 weeks of follow-up. Patients with T1DM more often were prescribed prokinetic agents, proton pump inhibitors, and anxiolytics, and had more GES implants compared with T2DM patients. These interventions had little 
positive impact on the symptoms in the T1DM subgroup, suggesting these patients had a refractory and end-stage condition. However, investigator ratings of gastroparesis severity improved in both diabetic groups at 48 weeks; it is possible that this divergence of clinician and patient ratings stemmed from decreases in the hospitalizations observed in T1DM patients. Nevertheless, future studies of investigational therapies of diabetic gastroparesis may need to consider differential responses in the two subtypes.

Our results showed no relationship between $\mathrm{HbAlc}$ levels and patient-reported GCSI scores or investigatorrated gastroparesis severity at 48 weeks. Furthermore, the decrease in GI symptoms reported by the T2DM patients occurred even though HbAlc values increased slightly over the 48 weeks. Thus, chronic glycemic control did not appear to influence GI symptoms in either group of diabetic patients with gastroparesis. Efforts for tighter glycemic control in patients with long-standing diabetes are important for many reasons, but these findings suggest that symptom reductions (particularly in T2DM patients) can occur without improved glucose control. Ongoing studies employing intensive insulin therapy will more rigorously determine if improved glycemia has additional symptom benefits. Although the presence of peripheral neuropathy was a discriminator of gastroparesis in T1DM vs T2DM on regression analysis, neuropathy did not relate to GCSI scores suggesting that peripheral and visceral complications of diabetes may not necessarily be linked. This finding also raises the possibility that gastroparesis in diabetes is not primarily neuropathic in origin, as suggested by histopathologic investigations performed by the GpCRC. ${ }^{26}$

Medication use over 48 weeks differed in the diabetic subtypes. Nearly half of the patients in the subgroups were receiving opioid agents at baseline, but T2DM patients more often were given new opiate prescriptions over 48 weeks of follow-up. Abdominal pain is the predominant symptom in $20 \%$ of gastroparesis patients, irrespective of etiology. ${ }^{4}$ The mechanisms for abdominal pain and the reasons for starting narcotics in T2DM patients are likely to be multifactorial; these could not be discerned from our analyses. In general, opiates slow gastric emptying and may worsen symptoms associated with gastroparesis. However, the reductions in GI symptom scores in the T2DM patients suggest that narcotics did not adversely affect these patients from an overall perspective. Many oral antidiabetic drugs such as metformin can cause nausea and vomiting. ${ }^{25}$ Unexpectedly, metformin use was actually associated with less vomiting in T2DM patients. Nevertheless, metformin intake should be considered among the causes of unexplained GI symptoms in T2DM patients.

Psychological dysfunction and quality of life are poor in patients with gastroparesis. ${ }^{11}$ These measures were equally poor in T1DM and T2DM patients at baseline, although more T1DM patients had severe anxiety. Psychological and quality of life parameters remained unchanged at 48 weeks of follow-up despite decreases in GI symptom scores in gastroparesis patients with T2DM. These findings suggest that GI symptom severity is not the only factor influencing either psychological distress or poor quality of life, at least in T2DM patients.

Gastric electrical stimulator therapy was employed more often for gastroparesis in T1DM patients compared to T2DM patients, but the T1DM group did not report a decrease in GI symptoms. Although differences did not reach significance, GES use also was higher over 48 weeks in the T2DM patients (change $=11 \%, p=0.06$ ). Gastric electrical stimulator therapy decreases nausea and vomiting in some but not all studies of diabetic gastroparesis. ${ }^{50}$ Future controlled investigations assessing GES efficacy in diabetic gastroparesis should be performed to contrast benefits in T1DM vs T2DM patients.

Our study had some limitations. Firstly, determination of diabetic subtype was dependent on subject report and review of the medical records by the investigator. Secondly, referral bias may have influenced the findings because the patients were referred to the tertiary motility centers of the GpCRC. Thus, our patients may not reflect typical patients managed in the community and they may have had clinical features that were unfavorable for symptom reductions over 48 weeks of follow-up. However, given the similarities in baseline GI symptoms in the T1DM and T2DM patients, it is likely referral bias was similar for both diabetic subtypes. Nevertheless, more T1DM patients had GES therapy which probably reflects the refractory nature of symptoms in this group. Thirdly, assessments of healthcare utilization did not include costs or address length of stay, outpatient visits, and missed work. Fourth, 48 weeks may be an inadequate time period to detect symptom score reductions in T1DM patients or differentiate resource utilization. The numbers of patients available for study at 48 weeks may have precluded some smaller differences not being detected; however, our study had $80 \%$ power to detect a minimal difference of 0.6 SD units in GCSI between the two subtypes. Finally, we had some concerns about nonsignificant trends to higher dropouts over 48 weeks of follow-up in the T1DM patients. Although several minor differences were observed in patients who did 
vs did not attend their 48 study visits, the lack of relation of 48-week visit attendance and GCSI scores, diabetes subtype, and hospitalizations confirmed that the different outcomes of T1DM and T2DM patients were not due to differential study compliance. We believe these limitations are countered by the significant strengths of the study including the large numbers of patients with gastroparesis and T1DM and T2DM, use of standardized tests and protocols and comprehensive collection of clinical, psychological, quality of life, and healthcare usage data.

In conclusion, our findings challenge several clinical axioms about gastroparesis in the two diabetic subtypes. Firstly, baseline GI symptoms associated with gastroparesis were remarkably similar in T1DM vs T2DM patients, even though T1DM patients had more severe gastric emptying delays and higher $\mathrm{HbAlc}$ values. These observations suggest that the presence of other gastric or extragastric pathogenic factors may mediate gastroparesis symptom severity. Secondly, symptoms associated with gastroparesis in both diabetic subtypes did not correlate with HbAlc levels or severity of gastroparesis; and last, after 48 weeks of follow-up, most GI symptom scores decreased only in T2DM patients even though T1DM patients showed increased medical and surgical treatment utilization. These similarities and differences in patients with T1DM and T2DM form a basis for further research to improve clinical outcomes with novel drugs, gastric stimulation parameters, and insulin dosing regimens for symptoms associated with gastroparesis.

\section{FUNDING}

This project received support from the National Institute of Diabetes and Digestive and Kidney Diseases (NIDDK) (grants U01DK073983, U01DK073975, U01DK073985, U01DK074007, U01DK073974, U01DK074008) as part of its funding of the Gastroparesis Clinical Research Consortium.

\section{DISCLOSURE}

None of the authors report any financial, professional, or personal conflicts of interest that are relevant to this manuscript.

\section{AUTHOR CONTRIBUTION}

KLK and WLH contributed toward study design, subject recruitment, data collection, data analysis, manuscript preparation, and approval of final manuscript version; KPY contributed toward study design, statistical analysis, manuscript preparation, and approval of final manuscript version; HPP, PJP, WJS, TLA, RWM, LAN, and IS contributed toward study design, subject recruitment, data collection, manuscript preparation, and approval of final manuscript version; JC-E and GF contributed toward study design, manuscript preparation, and approval of final manuscript version; JT and LL contributed toward study design, statistical analysis, manuscript preparation, and approval of final manuscript version. LM contributed toward manuscript preparation and approval of final manuscript version; FH contributed toward study design, grant administration, manuscript preparation, and approval of final manuscript.

\section{REFERENCES}

1 Camilleri M. Clinical practice. Diabetic gastroparesis. $N$ Engl I Med 2007; 356: 820-9.

2 Abell TL, Bernstein VK, Cutts T, Farrugia G, Forster J, Hasler WL, McCallum RW, Olden KW et al. Treatment of gastroparesis: a multidisciplinary clinical review. Neurogastroenterol Motil 2006; 18: 263-83.

3 Hasler WL, Wilson LA, Parkman HP, Nguyen L, Abell TL, Koch KL, Pasricha PJ, Snape WJ et al. Bloating in gastroparesis: severity, impact, and associated factors. Am I Gastroenterol 2011; 106: 1492-502.

4 Hasler WL, Wilson LA, Parkman HP, Koch KL, Abell TL, Nguyen L, Pasricha PJ, Snape WJ et al. Factors related to abdominal pain in gastroparesis: contrast to patients with predominant nausea and vomiting. Neurogastroenterol Motil 2013; 25: 427-38.
5 Tougas G, Eaker EY, Abell TL, Abrahamsson $\mathrm{H}$, Boivin $\mathrm{M}$, Chen $\mathrm{J}$, Hocking MP, Quigley EM et al. Assessment of gastric emptying using a low fat meal: establishment of international control values. $\mathrm{Am} J$ Gastroenterol 2000; 95: 1456-62.

6 Pasricha PJ, Colvin R, Yates K, Hasler WL, Abell TL, Unalp-Arida A, Nguyen L, Farrugia G et al. Characteristics of patients with chronic nausea and vomiting and normal gastric emptying. Clin Gastroenterol Hepatol 2011; 9: 567-76.

7 Janssen P, Harris MS, Jones M, Masaoka T, Farré R, Törnblom $\mathrm{H}$, Van Oudenhove L, Simrén M et al. The relation between symptom improvement and gastric emptying in the treatment of diabetic and idiopathic gastroparesis. Am I Gastroenterol 2013; 108: 1382-91.

8 Soykan I, Sivri B, Sarosiek I, Kiernan B, McCallum RW. Demography, clinical characteristics, psychological and abuse profiles, treatment, and longterm follow up of patients with gastroparesis. Dig Dis Sci 1998; 43: 2398 404.

9 Fraser RJ, Horowitz M, Maddox AF, Harding PE, Chatterton BE, Dent J. Hyperglycemia slows gastric emptying in type 1 (insulin-dependent) diabetes mellitus. Diabetologia 1990; 33: 675-80.

10 Hebbard GS, Samsom M, Sun WM, Dent J, Horowitz M. Hyperglycemia affects proximal gastric motor and sensory function during small intestinal triglyceride infusion. Am J Physiol 1996; 271: G814-9.

11 Hasler WL, Parkman HP, Wilson LA, Pasricha PJ, Koch KL, Abell TL, Snape WJ, Farrugia G et al. Psychological dysfunction is associated with symptom severity but not disease etiology or degree of gastric retention in patients with gastroparesis. Am I Gastroenterol 2010; 105: 2357-67. 
12 Kashyap P, Farrugia G. Diabetic gastroparesis: what we have learned and had to unlearn in the past 5 years. Gut 2010; 59: 1716-26.

13 Ramzan Z, Duffy F, Gomez J, Fisher RS, Parkman HP. Continuous glucose monitoring in gastroparesis. Dig Dis Sci 2011; 56: 2646-55.

14 Hyett B, Martinez FJ, Gill BM, Mehra S, Lembo A, Kelly CP, Leffler DA. Delayed radionucleotide gastric emptying studies predict morbidity in diabetics with symptoms of gastroparesis. Gastroenterology 2009; 137: 445-52.

15 Jung HK, Choung RS, Locke GR, Schleck CD, Zinsmeister AR, Szarka LA, Mullan B, Talley NJ. The incidence, prevalence, and outcomes of patients with gastroparesis in Olmstead County, Minnesota, from 1996 to 2006. Gastroenterology 2009; 136: 1225-33.

16 Jones KL, Russo A, Berry MK, Stevens JE, Wishart JM, Horowitz M. A longitudinal study of gastric emptying and upper gastrointestinal symptoms in patients with diabetes mellitus. Am I Med 2002; 113: 449-55.

17 Chang J, Rayner CK, Jones KL, Horowitz M. Prognosis of diabetic gastroparesis - a 25 year evaluation. Diabet Med 2013; 30: e185-8.

18 Pasricha PJ, Yates KP, Nguyen L, Clarke J, Abell TL, Farrugia G, Hasler $\mathrm{WL}$, Koch KL et al. Outcomes and factors associated with reduced symptoms in patients with pastroparesis. Gastroenterology 2015; 149: 1762-74. Doi:10.1053/j.gastro.2015.08.008.

19 Daneman D. Type 1 diabetes mellitus. Lancet 2006; 367: 847-58.

20 Lyssenko V, Jonsson A, Almgren P, Pulizzi N, Isomaa $\mathrm{B}$, Tuomi $\mathrm{T}$, Berglund $G$, Altshuler D. Clinical risk factors, DNA variants, and the development of type 2 diabetes. $N$ Engl I Med 2008; 359: 2220-32.

21 Horowitz M, Maddox AF, Wishart JM, Harding PE, Chatterton BE, Shearman DJ. Relationships between esophageal transit and solid and liquid gastric emptying in diabetes mellitus. Eur J Nuc Med 1991; 18: 229 34.

22 Keshavarzian A, Iber FL, Vaeth J. Gastric emptying in patients with insulin-requiring diabetes mellitus. Am I Gastroenterol 1987; 82: 29-35.

23 Horowitz M, Harding PE, Maddox AF, Wishart JM, Akkermans LM, Chatterton BE, Shearman DJ. Gastric and esophageal emptying in patients with type 2 (noninsulin-dependent) dia- betes mellitus. Diabetologia 1989, 32: $151-9$.

24 Choung RS, Locke GR, Schleck CD, Zinsmeister AR, Melton LJ, Talley NJ. Risk of gastroparesis in subjects with type 1 and type 2 diabetes in the general population. Am I Gastroenterol 2012; 107: 82-8.

25 Dickman R, Kislov J, Boaz M, Ron Y, Beniashvili Z, Raz I, Baigel I, Niv Y et al. Prevalence of symptoms suggestive of gastroparesis in a cohort of patients with diabetes mellitus. I Diab Comp 2013; 27: 376-9.

26 Kofod-Andersen K, Tarnow L. Prevalence of gastroparesis-related symptoms in an unselected cohort of patients with type 1 diabetes. I Diab Comp 2012; 26: 89-93.

27 Grover M, Farrugia G, Lurken MS, Bernard CE, Faussone-Pellegrini MS, Smyrk TC, Parkman HP, Abell TL et al. Cellular changes in diabetic and idiopathic gastroparesis. Gastroenterology 2011; 140: 1575-85.

28 Rentz AM, Kahrilas P, Stanghellini V, Tack J, Talley NJ, de la Loge C, Trudeau E, Dubois D et al. Development and psychometric evaluation of the patient assessment of upper gastrointestinal symptom severity index (PAGI-SYM) in patients with upper gastrointestinal disorders. Qual Life Res 2004; 13: 1737-49.

29 Revicki DA, Rentz AM, Dubois D, Kahrilas P, Stanghellini V, Talley NJ, Tack J. Development and validation of a patient-assessed gastroparesis symptom severity measure: the Gastroparesis Cardinal Symptom Index. Aliment Pharmacol Ther 2003; 18: 141-50.

30 Abell TL, Camilleri M, Donohoe K, Hasler WL, Lin HC, Maurer AH, McCallum RW, Nowak $\mathrm{T}$ et al. Consensus recommendations for gastric emptying scintigraphy. A joint report of the Society of Nuclear Medicine and The American Neurogastroenterology and Motility Society. Am I Gastroenterology 2008; 103: 753-63.

31 Richter P, Werner I, Heerlein A, Kraus A, Sauer H. On the validity of the Beck Depression Inventory. A review. Psychopathology 1998; 31: $160-8$.

32 Spielberger C, Gorsuch R, Lushene R. Manual for the State-Trait Anxiety Inventory. Palo Alto, CA: Consulting Psychologists Press, 1970.

33 De la Loge C, Trudeau E, Marquis P, Kahrilas P, Stanghellini V, Talley NJ,
Tack J, Revicki DA et al. Crosscultural development and validation of a patient self-administered questionnaire to assess quality of life in upper gastrointestinal disorders: the PAGI-QOL. Qual Life Res 2004; 13: 1751-62.

34 Ware JE, Kosinski M, Dewey JE. How to Score Version 2 of the SF-36 ${ }^{\circledR}$ Health Survey. Lincoln, RI: QualityMetric Incorporated, 2000.

35 Agresti A. Categorical Data Analysis, 2nd edn. Hoboken, NJ: John Wiley and Sons Inc, 2002.

36 Hosmer DW, Lemeshow S. Applied Logistic Regression, 2nd edn. Hoboken, NJ: John Wiley and Sons Inc, 2000.

37 McNemar Q. Note on the sampling error of the difference between correlated proportions or percentages. Psychometrika 1947; 12: 153-7.

38 Fleiss JL, Leven B, Paik MC. Statistical Methods for Rates and Proportions, 3rd edn. Hoboken, NJ: John Wiley and Sons, Inc., 2003: 374-80.

39 Draper N, Smith H. Applied Regression Analysis, 3rd edn. Hoboken, NJ: John Wiley and Sons Inc, 1998.

40 Rosenbaum PR. Observational Studies, 3rd edn. New York, NY: Springer, 2010.

41 Perneger TV. What's wrong with Bonferroni adjustments. BMJ 1998; 316: 1236-8.

42 Cao J, Zhang S. Multiple comparison procedures. JAMA 2014; 312: 543-4.

43 Bender R, Lange S. Adjusting for multiple testing: when and how? $I$ Clin Epidemiol 2001; 54: 343-9.

44 Parkman HP, Yates K, Hasler WL, Nguyen L, Pasricha PJ, Snape WJ, Farrugia G, Koch KL et al. Similarities and differences between diabetic and idiopathic gastroparesis. Clin Gastroenterol Hepatol 2011; 9: 1056-64.

45 Kumar A, Attaluri A, Hashmi S, Schulze KS, Rao SS. Visceral hypersensitivity and impaired accommodation in refractory diabetic gastroparesis. Neurogastroenterol Motil 2008; 20: 635-42.

46 Koch KL, Stern RM, Stewart WR, Vasey MW. Gastric emptying and gastric myoelectrical activity in patients with diabetic gastroparesis: effect of long-term domperidone treatment. Am I Gastroenterol 1989; 84: 1069-75.

47 Mearin F, Camilleri M, Malagelada JR. Pyloric dysfunction in diabetics with recurrent nausea and vomit- 
ing. Gastroenterology 1986; 90 : 1919-25.

48 Parkman HP, Yates KP, Hasler WL, Nguyan L, Pasricha PJ, Snape WJ, Farrugia G, Calles J et al. Dietary intake and nutritional deficiencies in patients with diabetic or idiopathic gastroparesis. Gastroenterology 2011; 141: 486-98.
49 Grover M, Bernard CE, Pasricha PJ, Lurken MS, Faussone-Pellegrini MS, Smyrk TC, Parkman HP, Abell TL et al. Clinical-histological associations in gastroparesis: results from the Gastroparesis Clinical Research Consortium. Neurogastroenterol Motil 2012; 24: 531-9.
50 Hasler WL. Methods of gastric electrical stimulation and pacing: a review of their benefits and mechanisms of action in gastroparesis and obesity. Neurogastroenterol Motil 2009; 21: 229-43.

\section{APPENDIX}

Members of the Gastroparesis Clinical Research Consortium:

Clinical Centers:

Johns Hopkins University, Baltimore, MD: Pankaj Jay Pasricha, MD (Principal Investigator); John Clarke, MD; Yale Kim, MHS MS.

Stanford University, Stanford, CA: Linda Nguyen, MD (Principal Investigator); Nighat Ullah, MD.

California Pacific Medical Center, San Francisco, $C A$ : William Snape, MD (Principal Investigator); Nata DeVole, RN; Mary Greene (2009-2011); Candice Lee; Courtney Ponsetto (2009-2010); Katerina Shetler, MD.

Temple University, Philadelphia, PA: Henry P. Parkman, MD (Principal Investigator); Steven Kantor; Vanessa Lytes, MSN CRNP; Amiya Palit, MD; Kellie Simmons, NP.

Texas Tech University Health Sciences Center, E1 Paso, TX: Richard W. McCallum, MD (Principal Investigator); Reza Hejazi, MD (2009-2011); Kathy Roeser (2008-2009); Irene Sarosiek, MD; Denise Vasquez, RN; Natalia Vega, RN.

University of Louisville, Louisville, KY: Thomas Abell, MD (Principal Investigator); Karen Beatty, RN; Lisa Hatter, RN; Ronna Howard; Lindsay Nowotny, PA-C.

University of Mississippi Medical Center, Jackson, MS: Shou Tang, MD (Principal Investigator); Om S Amin, MD (2010-2011); Olivia Henry, MS RD; Archana Kedar, MD; Valerie McNair (2008-2012); Susanne Pruett, RN (2007-2008); Margaret Smith, RN; Danielle Spree, RN (2007-2010).

University of Michigan, Ann Arbor, MI: William Hasler, MD (Principal Investigator); Michelle Castle (2008-2011); Radoslav Coleski, MD (2007-2009); Sophanara Wootten.

Wake Forest University, Winston-Salem, NC: Kenneth Koch, MD (Principal Investigator); Lynn Baxter; Anya Brown; Samantha Culler (2009-2012); Judy Hooker; Paula Stuart, PA.

\section{Resource Centers:}

Mayo Clinic College of Medicine (Pathology Analyses Center), Rochester, MN: Gianrico Farrugia, MD (Principal Investigator); Cheryl Bernard, Matthew Lurken.

MetroHealth Medical Center, Cleveland, OH: Jorge Calles-Escandon, MD.

National Institute of Diabetes, Digestive and Kidney Diseases, Bethesda, $M D$ : Jose Serrano, $\mathrm{MD}, \mathrm{PhD}$ (Program Official); Frank Hamilton, MD, MPH (Project Scientist); Steven James, MD; Rebecca Torrance, RN MSN; Rebekah Van Raaphorst, MPH.

Johns Hopkins University, Bloomberg School of Public Health (Data Coordinating Center), Baltimore, $M D$ : James Tonascia, $\mathrm{PhD}$ (Principal Investigator); Patricia Belt; Erin Corless Hallinan, MHS; Ryan Colvin, MPH (2007-2010); Michele Donithan, MHS; Mika Green, MA (2009-2012); Milana Isaacson; Wana Kim (2009-2011); Linda Lee, MD; Patrick May, MS; Laura Miriel; Alice Sternberg, ScM; Mark Van Natta, MHS; Ivana Vaughn, MPH; Laura Wilson, ScM; Katherine Yates, ScM.

\section{SUPPORTING INFORMATION}

Additional supporting information may be found in the online version of this article at the publisher's web site:

Data S1 Data acquisition-specific items characterized.

Table S1 Relation of severity to baseline metformin use in T2DM.

Table S2 Comparing relative odds of completing 48week testing in all diabetics.

Table S3 Relative odds of changes in 48-week outcomes comparing by baseline hemoglobin Alc and peripheral neuropathy.

Table S4 Relative odds of changes in 48-week outcomes comparing diabetics with gastroparesis by hemoglobin Alc change for T1DM and T2DM patients. 